\title{
gprofiler2 -- an R package for gene list functional enrichment analysis and namespace conversion toolset $\mathbf{g}:$ Profiler [version 1; peer review: 1 approved with reservations]
}

Liis Kolberg, Uku Raudvere, Ivan Kuzmin, Jaak Vilo, Hedi Peterson

Institute of Computer Science, University of Tartu, Tartu, Tartumaa, 51009, Estonia

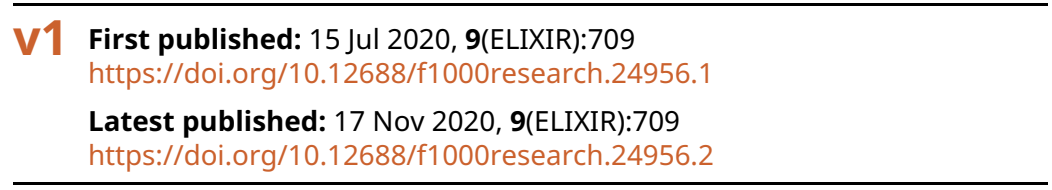

\section{Abstract}

g:Profiler (https://biit.cs.ut.ee/gprofiler) is a widely used gene list functional profiling and namespace conversion toolset that has been contributing to reproducible biological data analysis already since 2007. Here we introduce the accompanying R package, gprofiler2, developed to facilitate programmatic access to g:Profiler computations and databases via REST API. The gprofiler 2 package provides an easy-to-use functionality that enables researchers to incorporate functional enrichment analysis into automated analysis pipelines written in $\mathrm{R}$. The package also implements interactive visualisation methods to help to interpret the enrichment results and to illustrate them for publications. In addition, gprofiler 2 gives access to the versatile gene/protein identifier conversion functionality in $\mathrm{g}$ :Profiler enabling to map between hundreds of different identifier types or orthologous species. The gprofiler 2 package is freely available at the CRAN repository.

Keywords

g:Profiler, R package, functional enrichment analysis, identifier mapping, Gene Ontology, pathways

This article is included in the RPackage

gateway.

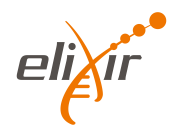

This article is included in the ELIXIR gateway.

\section{Open Peer Review}

Approval Status

1

2

version 2

(revision)

17 Nov 2020

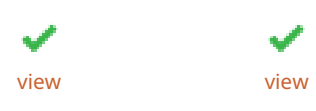

version 1

15 Jul 2020

1. Egon L. Willighagen (D), Maastricht

University, Maastricht, The Netherlands

2. Ewy Mathe (iD), National Center for

Advancing Translational Sciences/National

Institutes of Health, Bethesda, USA

Any reports and responses or comments on the article can be found at the end of the article. 
Corresponding author: Hedi Peterson (hedi.peterson@ut.ee)

Author roles: Kolberg L: Conceptualization, Formal Analysis, Methodology, Software, Visualization, Writing - Original Draft Preparation; Raudvere U: Conceptualization, Data Curation, Methodology, Software, Visualization, Writing - Review \& Editing; Kuzmin I:

Conceptualization, Methodology, Software, Visualization, Writing - Review \& Editing; Vilo J: Funding Acquisition, Resources, Writing Review \& Editing; Peterson H: Conceptualization, Funding Acquisition, Project Administration, Supervision, Writing - Review \& Editing

Competing interests: No competing interests were disclosed.

Grant information: This work was supported by the Estonian Research Council grants (IUT34-4;PSG59); the European Regional Development Fund for CoE of Estonian ICT research EXCITE projects; and Project No 2014-2020.4.01.16-0271, ELIXIR.

The funders had no role in study design, data collection and analysis, decision to publish, or preparation of the manuscript.

Copyright: (c) 2020 Kolberg L et al. This is an open access article distributed under the terms of the Creative Commons Attribution License, which permits unrestricted use, distribution, and reproduction in any medium, provided the original work is properly cited.

How to cite this article: Kolberg L, Raudvere U, Kuzmin I et al. gprofiler2 -- an R package for gene list functional enrichment analysis and namespace conversion toolset g:Profiler [version 1; peer review: 1 approved with reservations] F1000Research 2020, 9(ELIXIR):709 https://doi.org/10.12688/f1000research.24956.1

First published: 15 Jul 2020, 9(ELIXIR):709 https://doi.org/10.12688/f1000research.24956.1 


\section{Introduction}

Interpretation of gene lists is a key step in numerous biological data analysis workflows, such as differential gene expression analysis and co-expression clustering of RNA-seq or microarray data. Usually this involves associating these gene lists with previous knowledge from well curated data sources of biological processes and pathways. However, as the knowledge-bases are constantly changing, keeping the associations up to date requires careful data management. Handling numerous databases, especially when using different gene identifier types, can be a very time-consuming process for researchers.

g:Profiler (https://biit.cs.ut.ee/gprofiler) is a popular web toolset that helps to handle gene lists from various biological and biomedical studies of more than 600 species and strains, including vertebrates, plants, fungi, insects and parasites ${ }^{1,2}$. :Profiler's best known functionality is the over-representation analysis to identify significantly enriched biological functions and pathways obtained from well established data sources which include, among others, Gene Ontology $(\mathrm{GO})^{3}, \mathrm{KEGG}^{4}$ and Reactome ${ }^{5}$. The information about genes, identifier types and GO term associations in $\mathrm{g}$ :Profiler is mostly based on Ensembl databases ${ }^{6}$ including data from Ensembl Genomes, fungi, plants and metazoa specific versions of Ensembl. g:Profiler follows Ensembl's quarterly update cycle while keeping the access to previous data versions as archives for reproducibility. The parasite specific data is included from the WormBase ${ }^{7}$.

Providing users with fast and easy access has been the main goal of g:Profiler developers. Since 2007, g:Profiler has been in constant development and with the recent update in 2019 a new accompanying $\mathrm{R}$ package, gprofiler2, was developed ${ }^{8}$. The $\mathrm{R}$ package relies on the g:Profiler REST API requests providing an easy programmatic access to the same functionalities as in the web tool without performing heavy computations and mappings in $\mathrm{R}$. While there are other popular $\mathrm{R}$ packages for functional enrichment analysis, such as topGO $\mathrm{pand}^{9}$ clusterProfiler ${ }^{10}$, gprofiler2, unlike the others, provides access to numerous annotation data sources with a single query without requiring to download any of these sources to a local computer. Furthermore, the mapping between different gene identifiers is automatic and the input can be a mixed list of identifiers. g:Profiler's continuous development and flexibility of usage has been recognised by the European Life Science Infrastructure ELIXIR, which has selected it as one of its Recommended Interoperability Resources.

$\mathrm{g}:$ Profiler development team encourages and supports external tools and packages to use either gprofiler2 package or the public API to be part of their workflows. For example, RCAS Bioconductor package ${ }^{11}$ includes gprofiler2 for functional analysis of transcriptomic regions detected by different high-throughput experiments. Single-cell mapper package $(\mathrm{scMappR})^{12}$ analyses cell-type specific gene lists with gprofiler2. OmnipathR ${ }^{13}$ suggests using gprofiler2 for enrichment analysis of protein complexes. Gene Co-expression Network analysis pipeline (GWENA) uses gprofiler2 in their pipeline for functional enrichment of co-expressed gene modules. A Nextflow differential gene expression analysis pipeline includes gprofiler2 for pathway analysis.

Here we demonstrate how to conveniently incorporate the gprofiler $2 \mathrm{R}$ package into bioinformatics analysis pipelines using differential gene expression analysis as an example.

\section{Methods}

Implementation

Inherently, gprofiler $2^{8}$ is a collection of wrapper functions in $\mathrm{R}$ that simplify sending POST requests to the $\mathrm{g}$ : Profiler REST API using the RCurl package ${ }^{14}$. This means that all the annotation data sources and computations are centralised in a single well-maintained server and therefore the results from both the web tool and R package are guaranteed to be identical. Relying on the central API also simplifies the maintenance of the g:Profiler interfaces and enables the $\mathrm{R}$ users to get access to the most up-to-date data without having to download the heavy annotation data files to their own devices.

There are four main API wrapper functions in gprofiler2:

- gost for functional enrichment analysis

- gconvert for mapping gene identifiers between different namespaces

- gorth for mapping orthologous genes across species

- gsnpense for mapping SNP rs-IDs to chromosome positions, genes and variant effects. 
In addition to fetching the results from the API, gprofiler2 uses the packages ggplot $2^{15}$ and plotly ${ }^{16}$ to provide visualisations for enrichment results that are similar to the web tool ones. Using ggplot2 allows users to customise the visualisations by adding or removing graphical layers, and to adjust the quality of images for publication.

This article was written using R version 3.6.1 (2019-07-05) and gprofiler2 version 0.1.9.

Operation

The gprofiler $2 \mathrm{R}$ package is available from CRAN and works on R versions 3.5 and above. The package also includes a detailed vignette.

The package can be installed from CRAN:

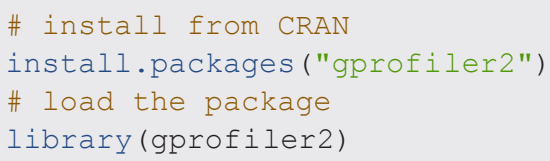

Input description

The most popular functionality of $\mathrm{g}$ :Profiler is functional enrichment analysis provided by the g:GOSt tool that performs over-representation analysis using hypergeometric test. This functionality is available in gprofiler2 under the function gost. The required inputs for this function are a vector of gene identifiers, query, and the name of the corresponding organism which is constructed by concatenating the first letter of the genus name and the specific epithet, e.g. hsapiens for human genes. The full list of supported species and strains, 641 in total, is available on the g:Profiler web page.

The query vector can include mixed types of gene/protein identifiers, SNP rs-IDs, chromosomal intervals or term IDs. Accepting a mixture of IDs is a unique feature that skips time-consuming manual steps of converting between different identifier types required by other functional enrichment tools. However, in case of analysing numeric identifiers (e.g. Entrez IDs) the user should specify the namespace using the numeric_ns parameter. The same description of input query and organism holds for the three other functions in gprofiler2.

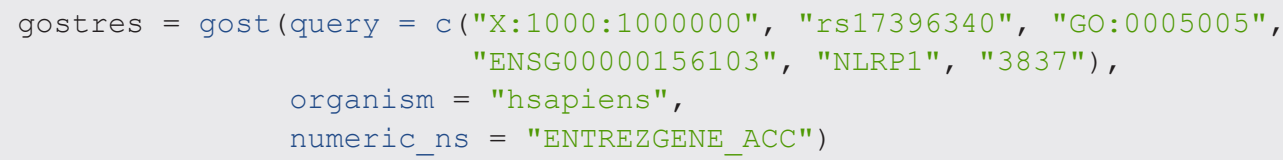

Several additional parameters in the gost function help to perform the analysis according to specific needs, including custom statistical options such as background definition, statistical significance threshold, method for multiple testing correction and testing for under-representation. Also, additional information like GO evidence codes and genes belonging to the intersection between the input list and the functional term is available.

\section{Annotation databases}

$\mathrm{g}$ :Profiler's in-house database includes only reliable annotation data sources that are regularly updated such as Gene Ontology $(\mathrm{GO})^{3}, \mathrm{KEGG}^{4}$, Reactome ${ }^{5}$, WikiPathways ${ }^{17}$, miRTarBase ${ }^{18}$, TRANSFAC ${ }^{19}$, Human Protein Atlas ${ }^{20}$, protein complexes from CORUM ${ }^{21}$ and Human Phenotype Ontology ${ }^{22}$. By default, all the data sources in $\mathrm{g}$ :Profiler database are used for the analysis in gprofiler2, but a specific selection can be made with the sources parameter of the gost function. In order to enable more flexibility, users can also use their own annotation data. The custom source can be uploaded in a Gene Matrix Transposed (GMT) file format. This feature is further described in the next section.

\section{Use case}

Differential gene expression analysis determines lists of genes that show changes in expression between different conditions, cell types, time points, etc. Functional enrichment analysis using the gprofiler 2 package ${ }^{8}$ helps to interpret these gene lists.

Here we demonstrate the main functionality of gprofiler 2 by following an analysis example from the existing RNA-seq Bioconductor workflow ${ }^{23}$ that uses the popular DESeq2 package ${ }^{24}$ for differential analysis. The example 
RNA-seq data are obtained from the airway package ${ }^{25}$ that comprises data from experiment where airway smooth muscle cells were treated with dexamethasone.

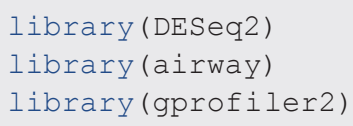

\section{Functional enrichment of differentially expressed genes}

First, we will detect the list of genes that are differentially regulated when stimulated with dexamethasone and then we will use the function gost from gprofiler 2 to find the biological functions and pathways that are significantly enriched in this gene set.

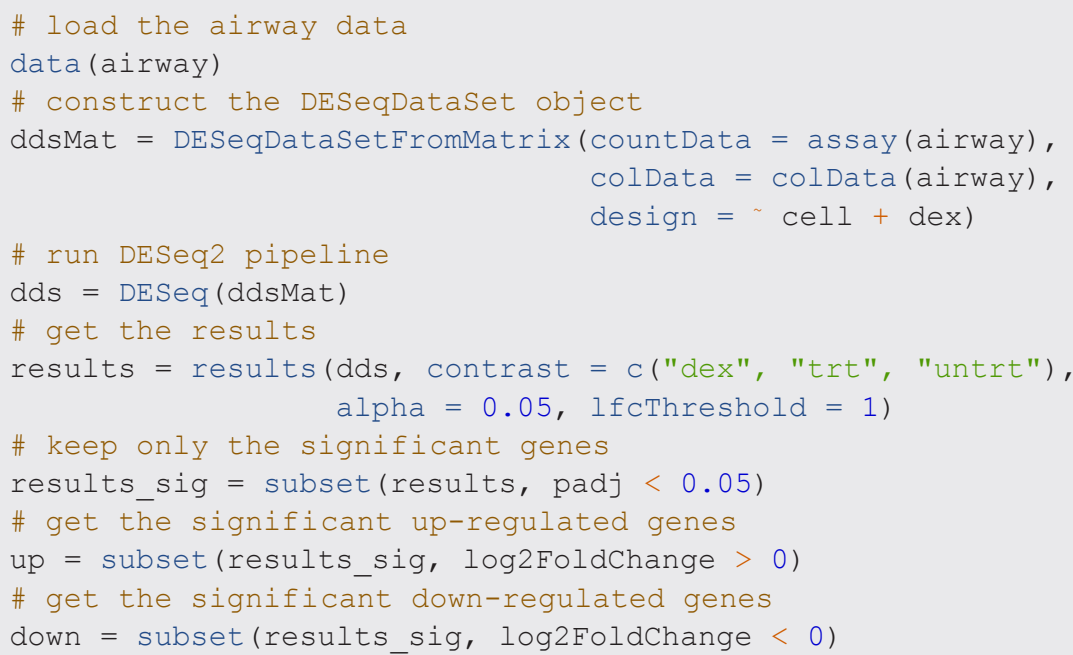

The output of the gost function is a named list where the element result includes a data frame with the enriched functions and related statistics; and the element meta includes relevant metadata for reproducing these results.

head (gp_up\$result)

\begin{tabular}{|c|c|c|c|c|}
\hline \#\# \# & query sic & P_value te & erm_size & equery_size intersection_size \\
\hline \#\# 1 & query_1 & TRUE $0.001 \overline{1} 14822$ & 9455 & 124 \\
\hline$\# \# 2$ & query_1 & TRUE 0.003848846 & 14 & 124 \\
\hline \#\# 3 & query_1 & TRUE 0.003848846 & 14 & 124 \\
\hline \#\# & query 1 & TRUE 0.006923191 & 16 & 124 \\
\hline 5 & query_1 & TRUE 0.006923191 & 16 & 124 \\
\hline \#\# & query_1 & TRUE 0.012886939 & 1024 & 124 \\
\hline \#\# \# & precision & term_id & source & term_name \\
\hline \#\# & 0.74193548 & $0.009730301 \mathrm{GO}: 0050896$ & $\mathrm{GO}: \mathrm{BP}$ & response to stimulus \\
\hline 2 & 0.03225806 & $0.285714286 \mathrm{GO}: 0010273$ & $\mathrm{GO}: \mathrm{BP}$ & detoxification of copper ion \\
\hline \#\# & 0.03225806 & 0.285714286 GO:1990169 & $\mathrm{GO}: \mathrm{BP}$ & stress response to copper ion \\
\hline \#\# & 0.03225806 & 0.250000000 GO:0097501 & $\mathrm{GO}: \mathrm{BP}$ & stress response to metal ion \\
\hline \#\# & 0.03225806 & 0.250000000 GO:0061687 & $\mathrm{GO}: \mathrm{BP}$ & detoxification of inorganic compound \\
\hline 6 & 0.16935484 & $0.020507812 \mathrm{GO}: 0009725$ & $\mathrm{GO}: \mathrm{BP}$ & response to hormone \\
\hline & effective_c & domain_size source_order & & parents \\
\hline \#\# & & $17906 \quad 15742$ & & GO : 0008150 \\
\hline \#\# 2 & & 17906 & $6 \mathrm{GO}: 006$ & 1687, GO: 1990169 \\
\hline
\end{tabular}




$\begin{array}{llrl}\text { \#\# } & 3 & 17906 & 29137 \mathrm{GO}: 0046688, \mathrm{GO}: 0097501 \\ \# \# & 4 & 22329 \mathrm{GO}: 0006950, \mathrm{GO}: 0010038 \\ \text { \#\# } 5 & 17906 & 18680 & \mathrm{GO}: 0098754 \\ \# \# & 6 & 17906 & 4118 \mathrm{GO}: 0009719, \mathrm{GO}: 0010033\end{array}$

Accounting for the order of genes in enrichment analysis

For cases where the list of interesting genes can be ranked by some biologically meaningful measure, such as P-value or fold change in differential analysis, g:Profiler provides an ordered query option that takes the ranking into account when performing enrichment tests. The testing is then performed iteratively, starting from the first gene and sequentially adding genes one by one. For every term, the smallest enrichment P-value is reported along with the corresponding gene list size. Consequently, for different terms the query size can vary, especially as the broader terms can be enriched for larger lists only. This option is very similar to the idea of the GSEA analysis method $^{26}$.

For example, to perform ordered query using gprofiler2 we first rearrange the list of up-regulated genes based on the $\log _{2}$ fold change values so that the first gene in the list has the highest value. Next we use this ordered list as a query in the gost function and set the parameter ordered_query = TRUE.

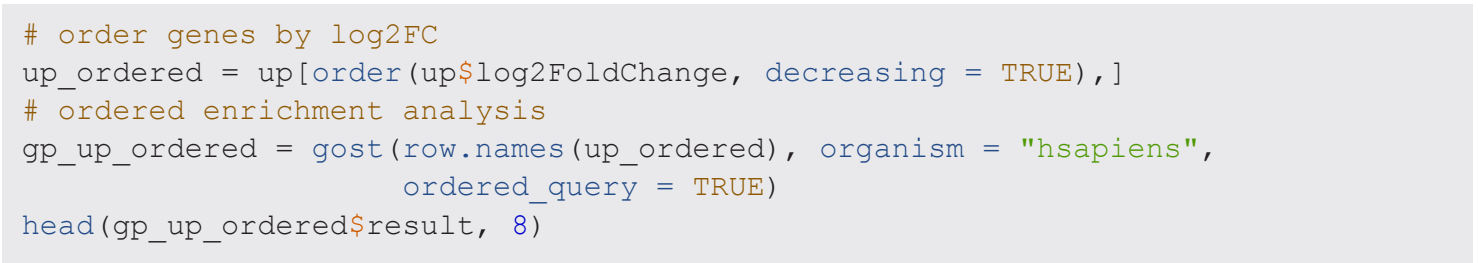

\begin{tabular}{|c|c|c|c|c|c|}
\hline \# \# & & query sic & nificant & P_value $t$ & term_size query_size intersection_size \\
\hline \#\# & 1 & query_1 & TRUE 0 & $0.0006 \overline{6} 17979$ & $\begin{array}{lll}- & 14 & -\end{array}$ \\
\hline \# \# & 2 & query_1 & TRUE 0 . & 0.0006617979 & 14 \\
\hline \#\# & 3 & query_1 & TRUE 0 . & 0.0011951178 & 16 \\
\hline \#\# & 4 & query_1 & TRUE 0. & 0.0011951178 & 16 \\
\hline \#\# & 5 & query_1 & TRUE 0. & 0.0047065333 & 22 \\
\hline \#\# & 6 & query_1 & TRUE 0. & 0.0080566224 & 25 \\
\hline \#\# & 7 & query_1 & TRUE 0. & .0132789677 & 106 \\
\hline \#\# & 8 & query_1 & TRUE 0 . & .0159695023 & 109 \\
\hline \#\# & & precision & recall & Lerm_id $\mathrm{s}$ & term_name \\
\hline \#\# & 1 & 0.05000000 & 0.28571429 & $9 \mathrm{GO}: 1990 \overline{1} 69$ & stress response to copper ion \\
\hline \#\# & 2 & 0.05000000 & 0.28571429 & GO: 0010273 & detoxification of copper ion \\
\hline \# \# & 3 & 0.05000000 & 0.25000000 & GO:0097501 & $\mathrm{GO}: \mathrm{BP} \quad$ stress response to metal ion \\
\hline \#\# & 4 & 0.05000000 & 0.25000000 & $\mathrm{GO}: 0061687$ & $\mathrm{GO}$ : $\mathrm{BP}$ detoxification of inorganic compound \\
\hline \#\# & 5 & 0.05000000 & 0.18181818 & GO:0071294 & $\mathrm{GO}: \mathrm{BP} \quad$ cellular response to zinc ion \\
\hline \#\# & 6 & 0.05000000 & 0.16000000 & $\mathrm{GO}: 0071280$ & cellular response to copper ion \\
\hline \#\# & 7 & 0.05833333 & 0.06603774 & GO: 0003300 & cardiac muscle hypertrophy \\
\hline \#\# & 8 & 0.05833333 & 0.06422018 & GO : 0014897 & striated muscle hypertrophy \\
\hline \#\# & & effective_c & domain_size & source_order & $r \quad$ parents \\
\hline \#\# & 1 & & $\overline{1} 7906$ & $6 \quad 29137$ & $7 \mathrm{GO}: 0046688, \mathrm{GO}: 0097501$ \\
\hline \#\# & 2 & & 17906 & 4576 & 6 GO:0061687, GO:1990169 \\
\hline \#\# & 3 & & 17906 & 22329 & $9 \mathrm{GO}: 0006950, \mathrm{GO}: 0010038$ \\
\hline \#\# & 4 & & 17906 & 18680 & $0 \quad$ GO: 0098754 \\
\hline \#\# & 5 & & 17906 & 19684 & $4 \mathrm{GO}: 0010043, \mathrm{GO}: 0071248$ \\
\hline \#\# & 6 & & 17906 & 19670 & $0 \mathrm{GO}: 0046688, \mathrm{GO}: 0071248$ \\
\hline \#\# & 7 & & 17906 & 1905 & $5 \quad$ GO $: 0014897$ \\
\hline \#\# & 8 & & 17906 & 5377 & $\mathrm{GO}: 0014896$ \\
\hline
\end{tabular}

The resulting data frame is in the same format as shown previously. Only the size of the query in the table can vary as the algorithm detects the most significant cutting point from the input gene list considering every function separately. 
Visualisation of functional enrichment results

Different visualisations are useful to summarise and interpret functional enrichment results. With the recent update, $\mathrm{g}$ :Profiler introduced an alternative way for visualising functional terms, a Manhattan plot. On this plot, the $\mathrm{x}$-axis shows the terms and y-axis shows the enrichment P-values on $-\log _{10}$ scale. Each circle on this plot corresponds to a single term. The circles are colored according to the annotation source and size-scaled according to the total number of genes annotated to the corresponding term. The locations on the $\mathrm{x}$-axis are always fixed and ordered in a way that the terms from the same GO subtree are located closer to each other. This helps to highlight different enriched GO sub-branches as they form peaks in the Manhattan plot and makes plots from different queries easily comparable. For the same reason, by default the values on the y-axis are capped to a maximum value of 16 that corresponds to P-value less than $10^{-16}$. The same default threshold is also used in the statistical tests in R. This selection can be switched off to show the P-values in a wider scale range.

Interactive graphs are common in web tools and therefore the Manhattan plot in g:Profiler web interface also provides several interactive features to facilitate data exploration and enables to export the visualisations as high-quality image files. Mimicking the g:Profiler web interface, the Manhattan plot in gprofiler2 is implemented in the function gostplot that uses the resulting object from the gost function as an input. As a unique feature, compared to other similar packages, the parameter interactive enables to switch between interactive plotly graph for browsing or static ggplot graph for saving as an image file. The parameter capped enables to turn off the upper limit of y-axis.

$$
\mathrm{p}=\text { gostplot (gp_up, interactive = TRUE) }
$$

After exploring the interactive graph and deciding on the story to tell about the results, the user can compose a publishable figure that highlights the most important terms using the function publish_gostplot and defining the relevant terms in the parameter highlight terms. The chosen terms are indicated with numbers on the plot and corresponding statistics are shown in the table below the Manhattan plot. For example, the enrichment results for up-regulated genes are shown in Figure 1. The Manhattan plot can be saved as an image file (PNG, PDF, JPEG, etc) specified by the $\mathrm{f}$ i lename parameter.

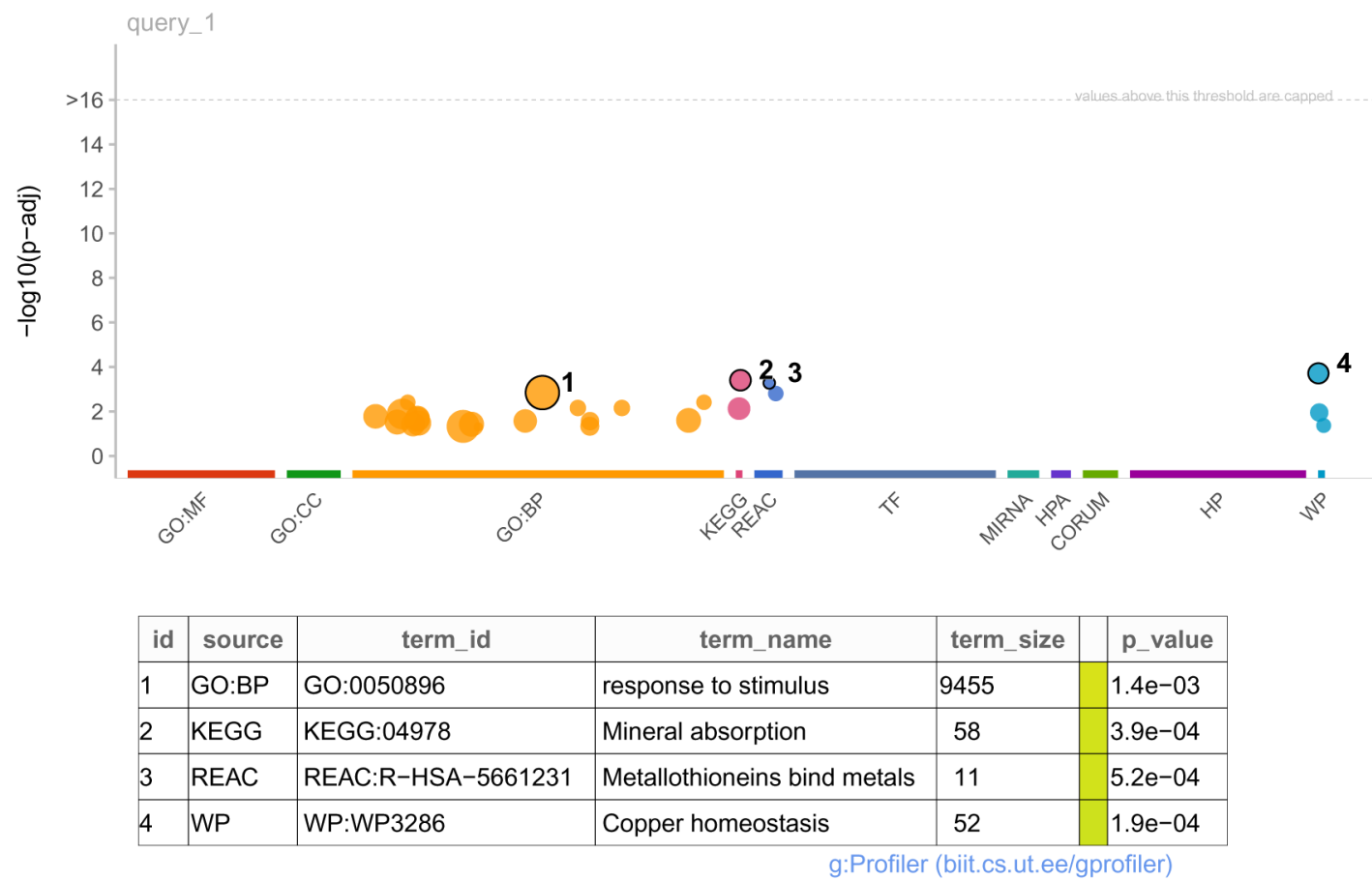

Figure 1. Manhattan plot of g:Profiler enrichment results. 


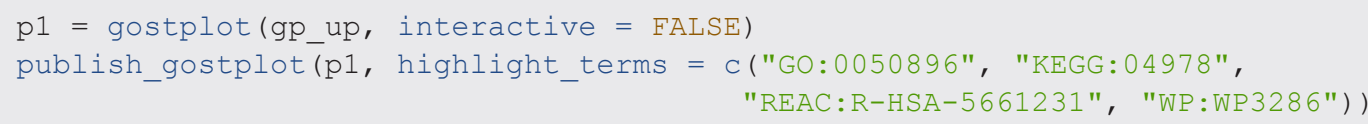

As the resulting plot is a standard ggplot object, it is easy to further customise the graphs by adding graphical layers or textual annotations.

Analysing multiple gene lists

Above we were analysing the up- and down-regulated gene lists separately, but the gost function also works with a (named) list of multiple gene vectors that enables to keep all the results in a single object and to easily compare different groups.

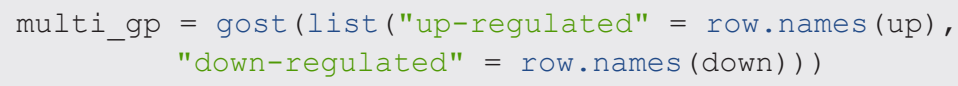

In this case, the resultant data frame is in a so-called "long format" where the column query includes the names of corresponding input vectors to differentiate between them. Alternative option is to set multi_query = TRUE which, in case of multiple gene lists, returns results as a comparison table in a "wide format". That is, the rows are concatenated by terms and query statistics are shown in cells as vectors, e.g. the p_values column includes a vector of corresponding P-values from all the input queries, even the insignificant ones.

Results from multiple gene lists can also be used for plotting. The function gostplot detects the case of multiple queries and plots the Manhattan plots under each other for comparison. The example enrichment results are shown in Figure 2.
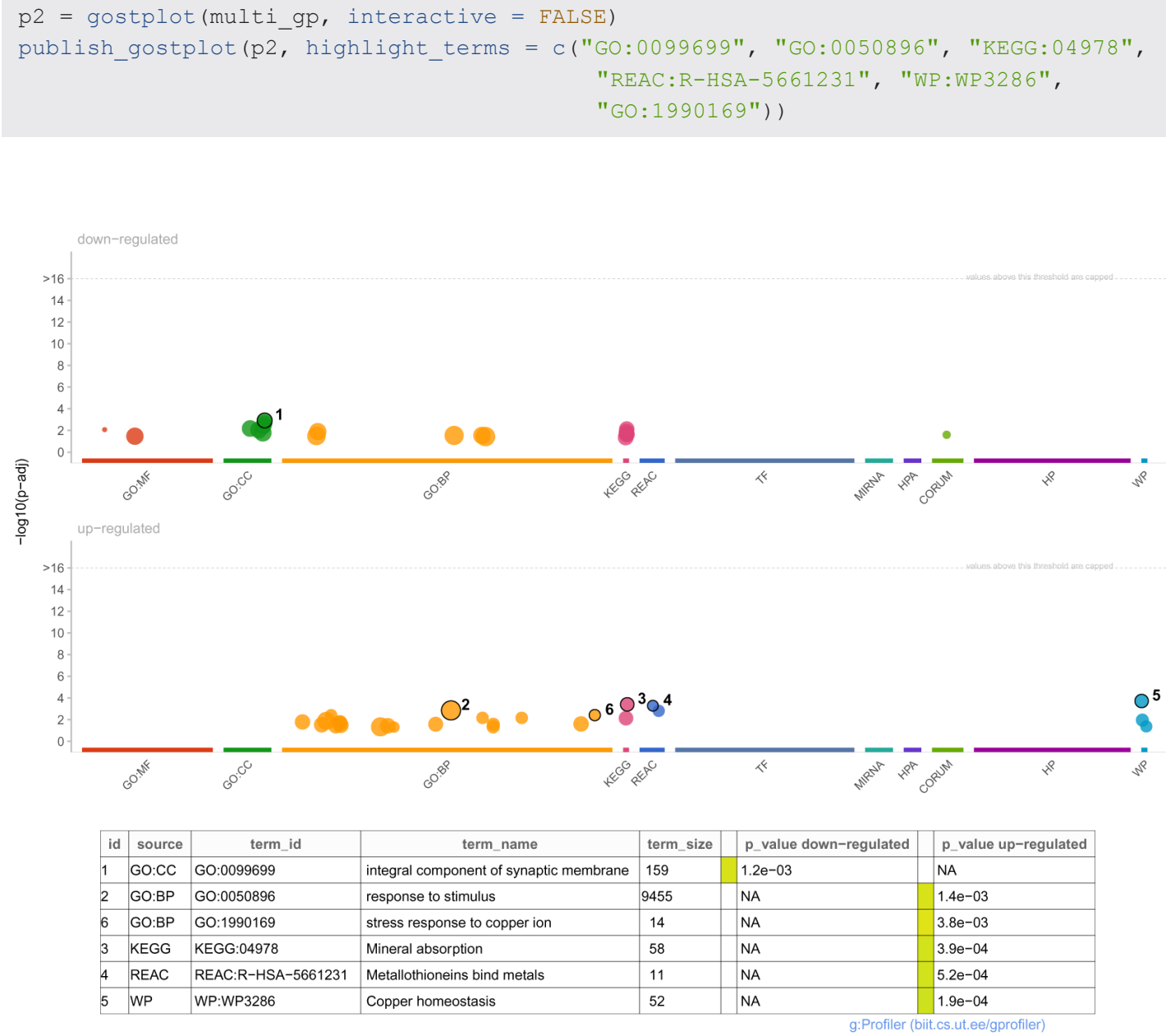

Figure 2. Visualisation of g:Profiler enrichment results to compare multiple gene lists. 
Sending analysis from $\mathrm{R}$ to $\mathrm{g}$ :Profiler web interface

The same enrichment results can also be viewed in the g:Profiler web tool. The user can generate a dedicated short-link by setting the parameter as_short_link = TRUE in the gost function which then returns the short-link to g:Profiler web tool instead of a data frame. This is a useful feature for sharing the results quickly with colleagues or to accompany a publication without the peers having to run the full analysis code in R.

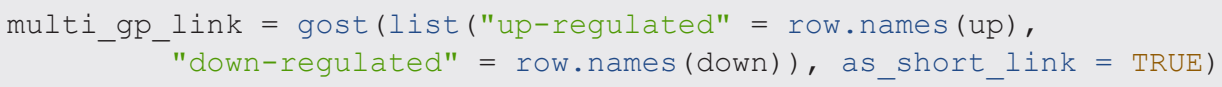

In this case, the variable multi_gp_link is a character string that corresponds to a stable short-link to these enrichment results: https://biit.cs.ut.ee/gplink/l/0wgtcERnQT.

Mapping between gene identifiers with gorth

Another common but tedious task in handling gene lists is mapping between different identifiers. The function gconvert helps to easily solve this issue and translates the given input identifiers to some other user defined namespace together with gene names and descriptions. The function is able to map between at least 30 different namespaces for more than 190 species. All available namespaces for different organisms are listed on the g:Profiler page.

As an example we will convert the Ensembl IDs in our differential expression results to numeric Entrez IDs with gconvert. The function takes a vector of gene identifiers as an input and returns a data frame that includes a column with target identifiers together with the names and descriptions for the input genes.
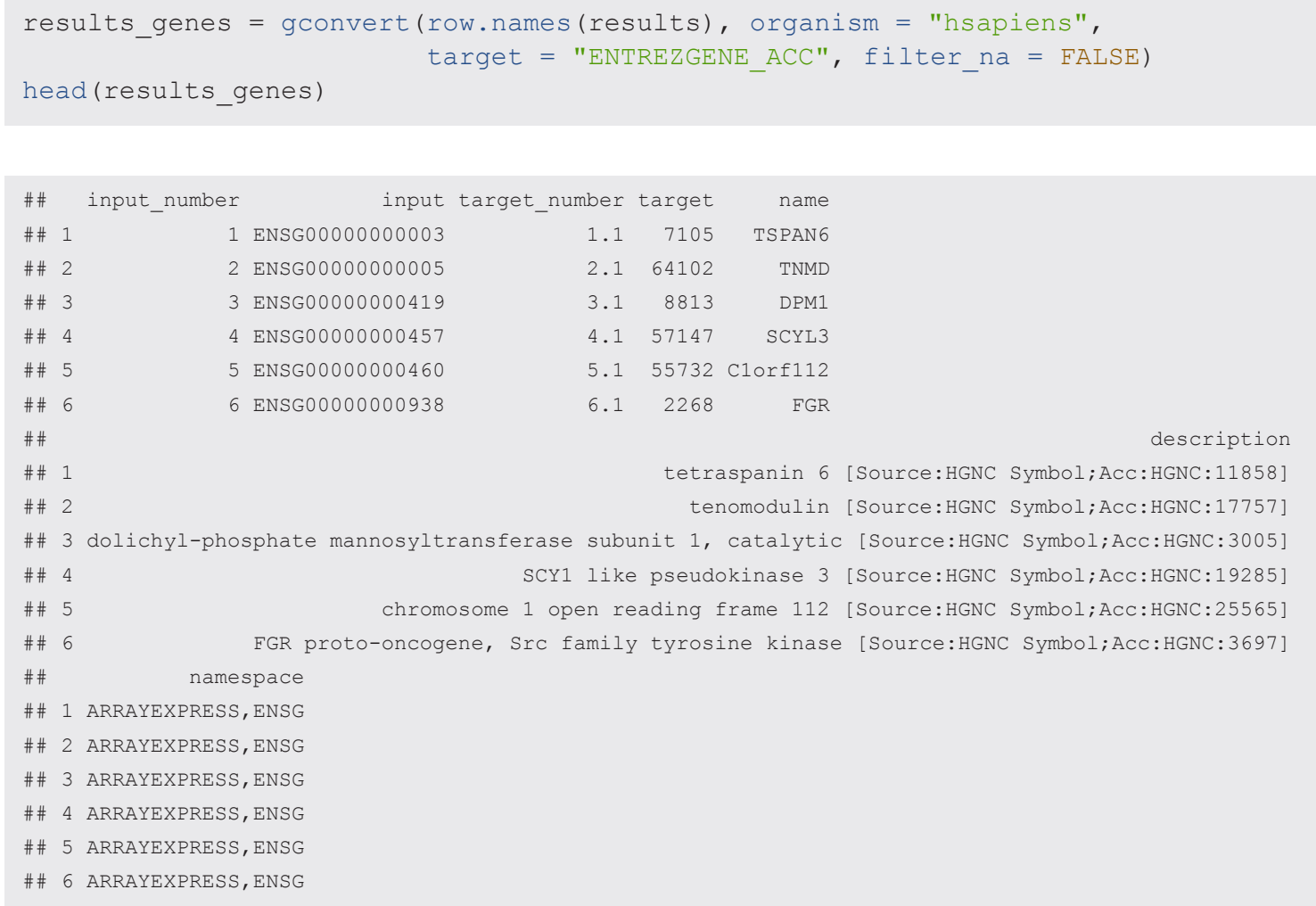

The users can add this information to the differential expression results data frame and save it to a tab separated text file to include as a supplementary file in their article, for example.

results_df $=$ as.data.frame (results)

results_df $\$$ Ensembl_id = row.names (results_df)

results_df $=$ results_df [order(results_df $\$ \overline{p a d j})$, 


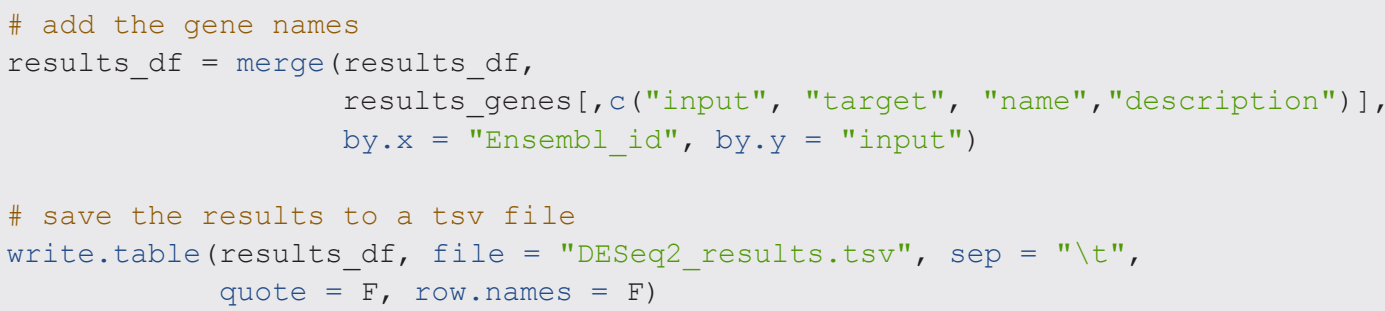

\section{Using custom annotations}

While g:Profiler enables to analyse genes from numerous organisms using high-quality annotation databases, there is still a need for custom data functionality for researchers interested in non-model organisms, that are not annotated in the Ensembl database, or in some specific, not so widespread annotation resource. In g:Profiler, this is solved by enabling users to upload custom annotation files in the GMT file format, which is essentially a tab delimited text file where every row describes a function by its identifier, description, and the genes annotated in this function. Here it is important to note that in case of custom annotation files, all the identifiers not present in the GMT file will be ignored in the analysis.

For example, to use the gene-disease association data from the DisGeNET database ${ }^{27}$ for enrichment analysis, the user can upload the GMT file in R using the upload_GMT_file function that returns a unique token for the file which can then be used as a value for the organism argument in the gost function.

First, we use R utility function download.file to download an annotation GMT file from DisGeNET into a file in the working directory and name it "DisGeNET.gmt".

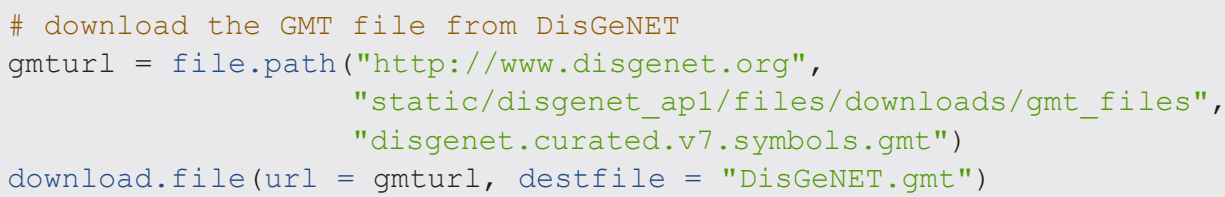

Now, when we have the file in our local environment, we can upload it to g:Profiler with the upload GMT file function.

token = upload_GMT_file(gmtfile = "DisGeNET.gmt")

\# save this token to your notes for enrichment analysis

The result of this upload is a unique token (in this case "gp_goJy_Ej2J_rPc") which should be saved by the user for future use. In order to find the enriched diseases in our gene list, we will use the token as a value for the organism in the gost function. As the DisGeNET database file includes gene symbols and not Ensembl identifiers, we first use gconvert to map our Ensembl IDs to gene names and use these as the input for the enrichment analysis.

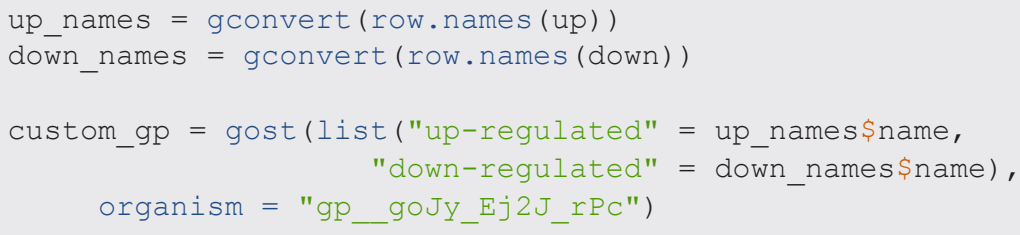

The custom data source results can also be plotted using the Manhattan plots (Figure 3).

$\mathrm{p}=$ gostplot (custom_gp, interactive = FALSE, pal = list("DisGeNET" = "salmon")) $\mathrm{pp}=$ publish_gostplot(p, highlight_terms = c ("C0011603", "C0014175"))

As the gprofiler2 $\mathrm{R}$ package and the web tool are in sync, this token will also work for the analysis in the web tool and can be inserted under the section "Bring your own data (Custom GMT)". And vice versa, the token obtained from the web tool will work in the $\mathrm{R}$ package without uploading the data again. Thus, in order to analyse multiple gene lists with the same data source, the user needs to upload the file only once and can use the 


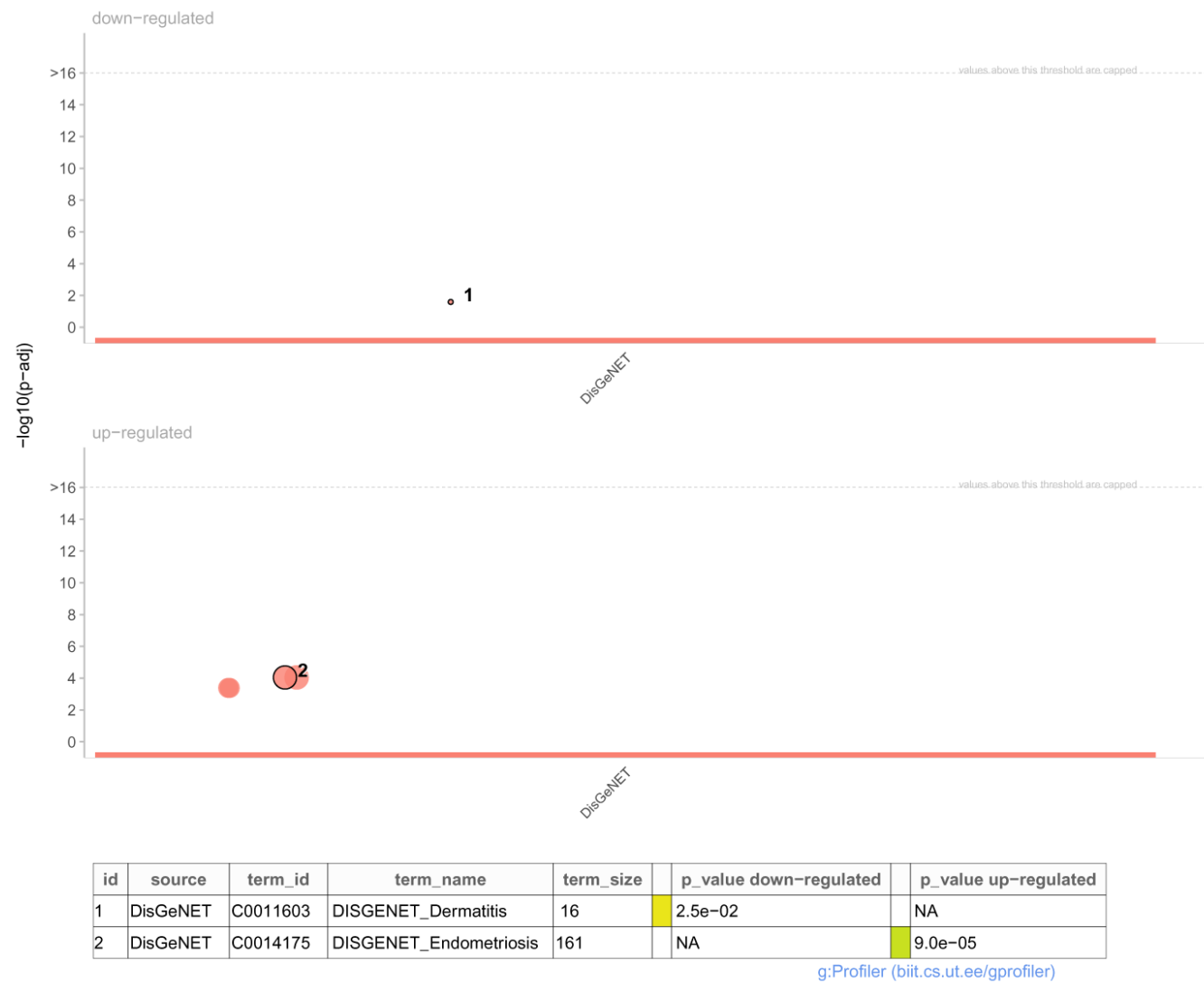

Figure 3. Manhattan plot of g:Profiler enrichment results using DisGeNET database loaded from a custom GMT file.

given token from then on. Furthermore, analysing multiple custom sources at once is enabled with the upload of a ZIP archive that includes multiple GMT files. GMT file names are used as the names for the data sources in the results and colored independently in the Manhattan plot.

\section{Mapping orthologous genes with gorth}

Sometimes, in order to further investigate the interesting set of differential genes in human, researchers need to perform additional experiments on model organisms such as mice. This requires finding the corresponding orthologs of these interesting genes from other species. Another use for orthologous genes is the possibility to transfer the extensive knowledge from well studied organisms to less studied species.

Mapping orthologous genes between species in $\mathrm{g}$ :Profiler is enabled by the $\mathrm{g}$ :Orth tool and in the gprofiler2 package the access is wrapped into the function gorth. The function works very similarly to gconvert, only in this case the user has to define corresponding source organism and target organism. For example, the following code maps the detected up-regulated gene identifiers to corresponding mice genes.

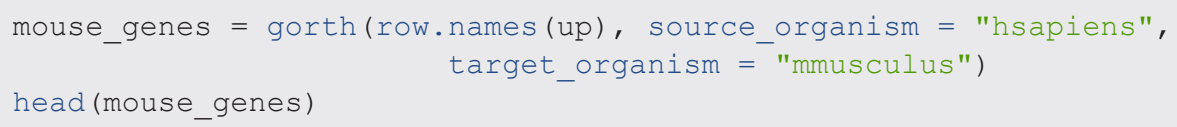

\begin{tabular}{|c|c|c|c|c|c|}
\hline \# \# & input_number & input & input_ensg & ensg_number & ortholog_name \\
\hline \# \# & $\begin{array}{lll}1 & - & 1\end{array}$ & ENSG00000035664 & ENSG0000003 5664 & 1.1 .1 & Dapk \\
\hline \# \# & 2 & ENSG00000046653 & ENSG00000046653 & 2.1 .1 & Gpm \\
\hline \# \# & 3 & ENSG00000060718 & ENSG00000060718 & 3.1 .1 & Col11a \\
\hline 4 & 4 & ENSG00000068383 & ENSG00000068383 & $4.1 \cdot 1$ & Inpp \\
\hline 5 & 5 & ENSG00000068831 & ENSG00000068831 & 5.1 .1 & Rasg \\
\hline 6 & 6 & ENSG00000070404 & ENSG00000070404 & 6.1 .1 & Fs \\
\hline
\end{tabular}




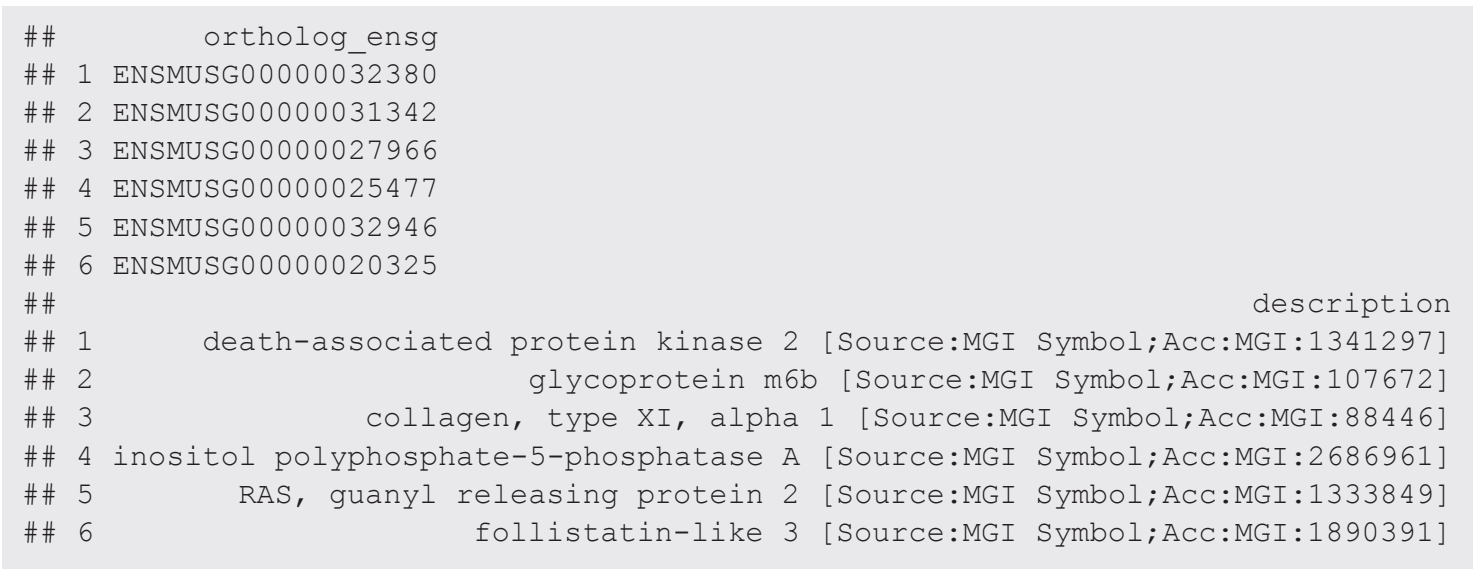

This function returns a data frame that includes the input and target identifiers, and also the ortholog names and descriptions.

Integrating with external tools for visualisations

Plots from enrichplot. Since the output of the gost function is stored in a standard data frame format, it is easy to alter it for custom visualisations using ggplot2, enrichplot ${ }^{28}$, clusterProfiler ${ }^{10}$ or any other similar package. Here we demonstrate how to convert the results from multiple gene lists into enrichResult and compareClusterResult objects required by the visualisations methods implemented in the enrichplot package. Similar approach also works for a single query.

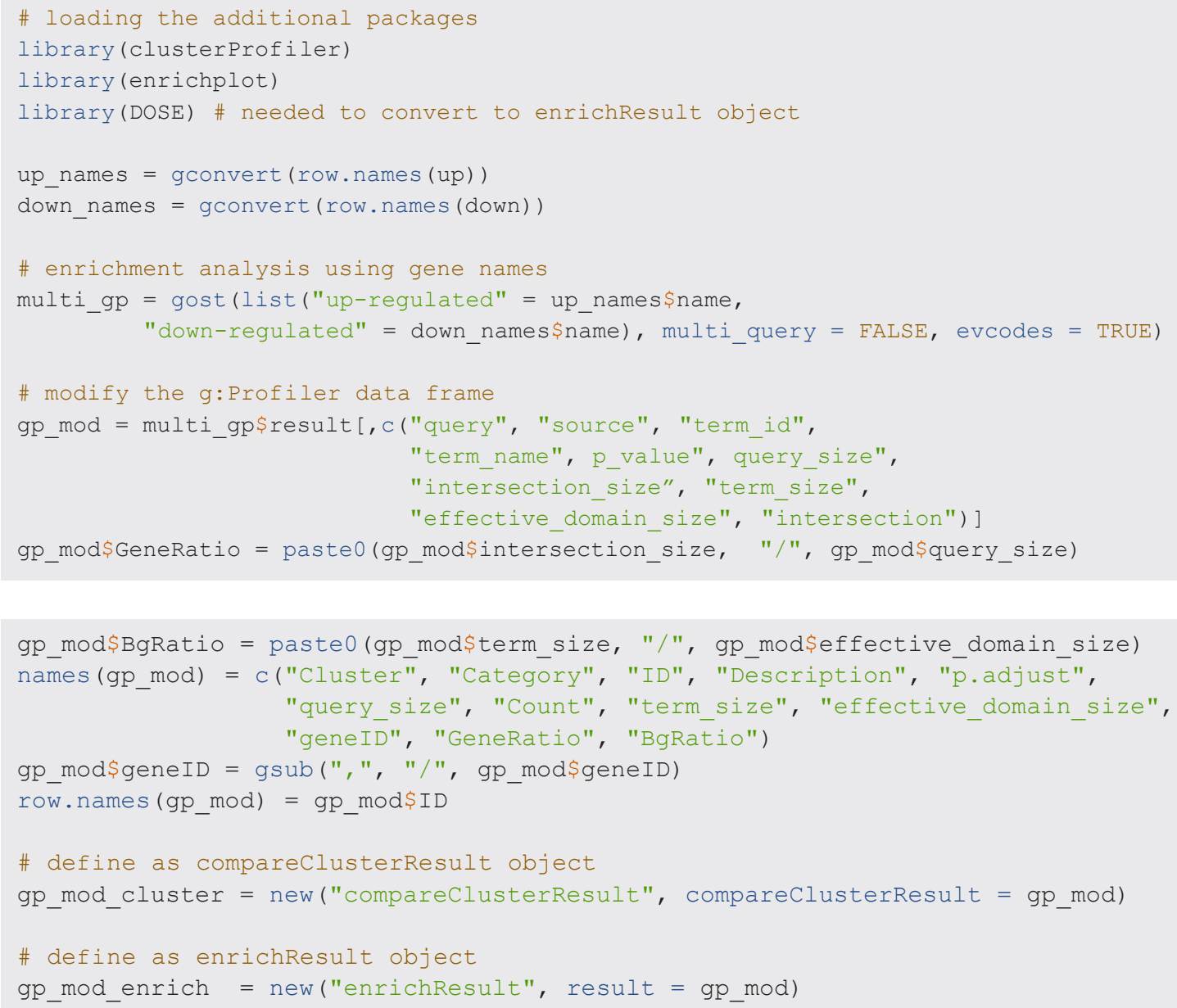


After creating an instance of the enrichResult or compareclusterResult (for multiple gene lists) class from the gost result, this object can be used as an input for the visualisation functions from enrichplot and clusterprofiler that are suitable for over-representation analysis such as dotplot, barplot, cnetplot, upsetplot, emapplot, etc. Figure 4 shows the dot plot for results in a compareclusterResult object.

$$
\text { enrichplot: :dotplot(gp_mod_cluster) }
$$

As these plots are ggplot objects, using ggplot2 layers allows further customisation of the visualisations as shown in Figure 5.

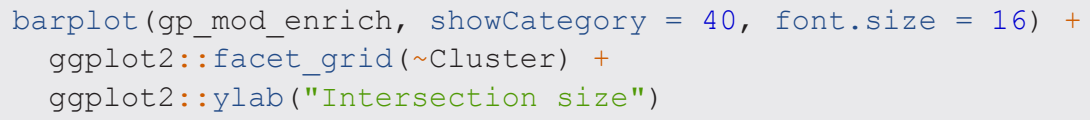

In order to use the browseKEGG function to open KEGG pathway browser, the pathway IDs should be transformed according to the organism. In case of human pathways, the prefix KEGG should be replaced with $h s a$. The full list of organisms and their prefixes is available from the KEGG home page.

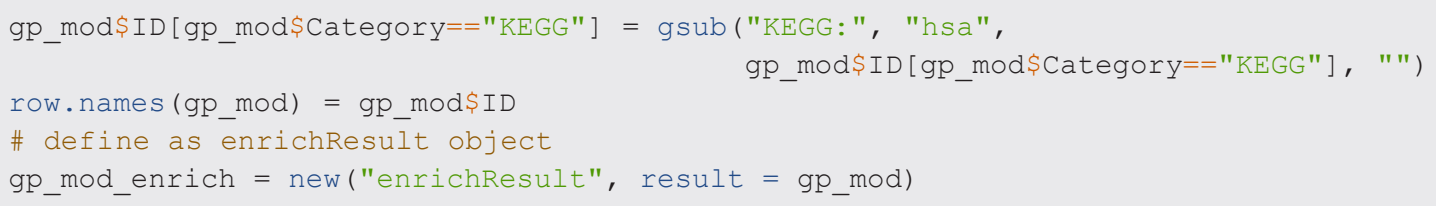

This command will open the KEGG browser page for the pathway Inflammatory mediator regulation of TRP channels.

\section{Using g:Profiler results in EnricmentMap}

The functional enrichment results from the gost function can be modified in order to save them into a Generic Enrichment Map (GEM) file format that is compatible with the EnrichmentMap application in Cytoscape $^{29}$. This app helps to visualise enrichment results as a highly customisable network where nodes represent enriched terms and edges represent their mutual overlap.

In case of a single query, the GEM file can be generated with the following lines of code. The parameter value evcodes = TRUE is important for obtaining the intersection column with corresponding gene IDs

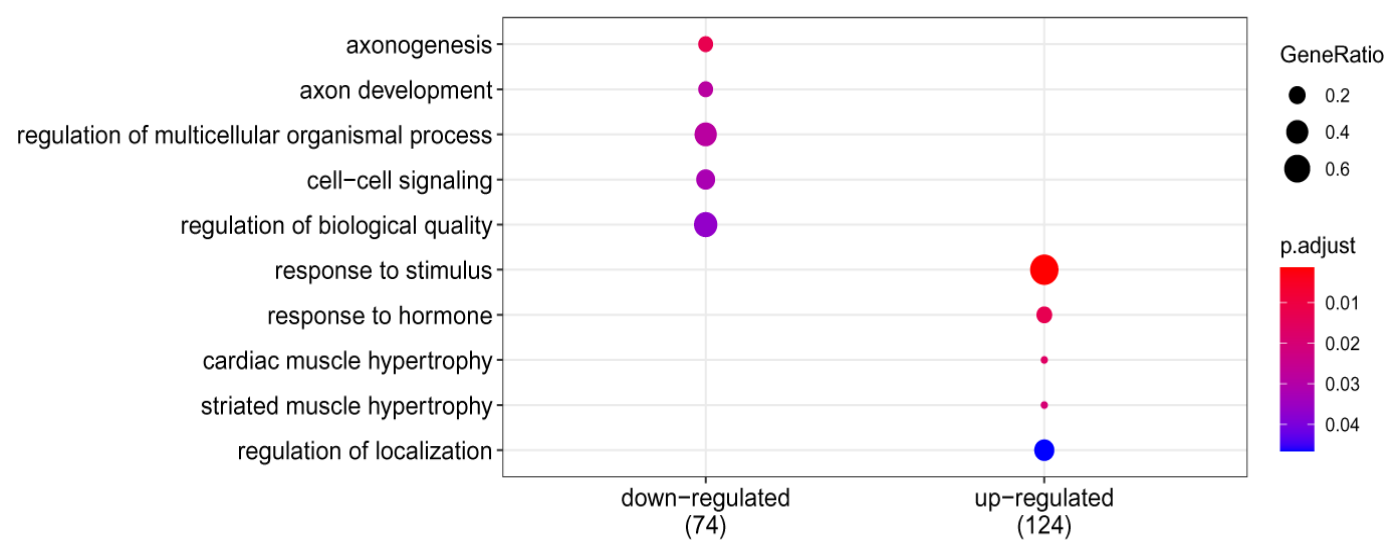

Figure 4. Dot plot of g:Profiler enrichment results using enrichplot. 


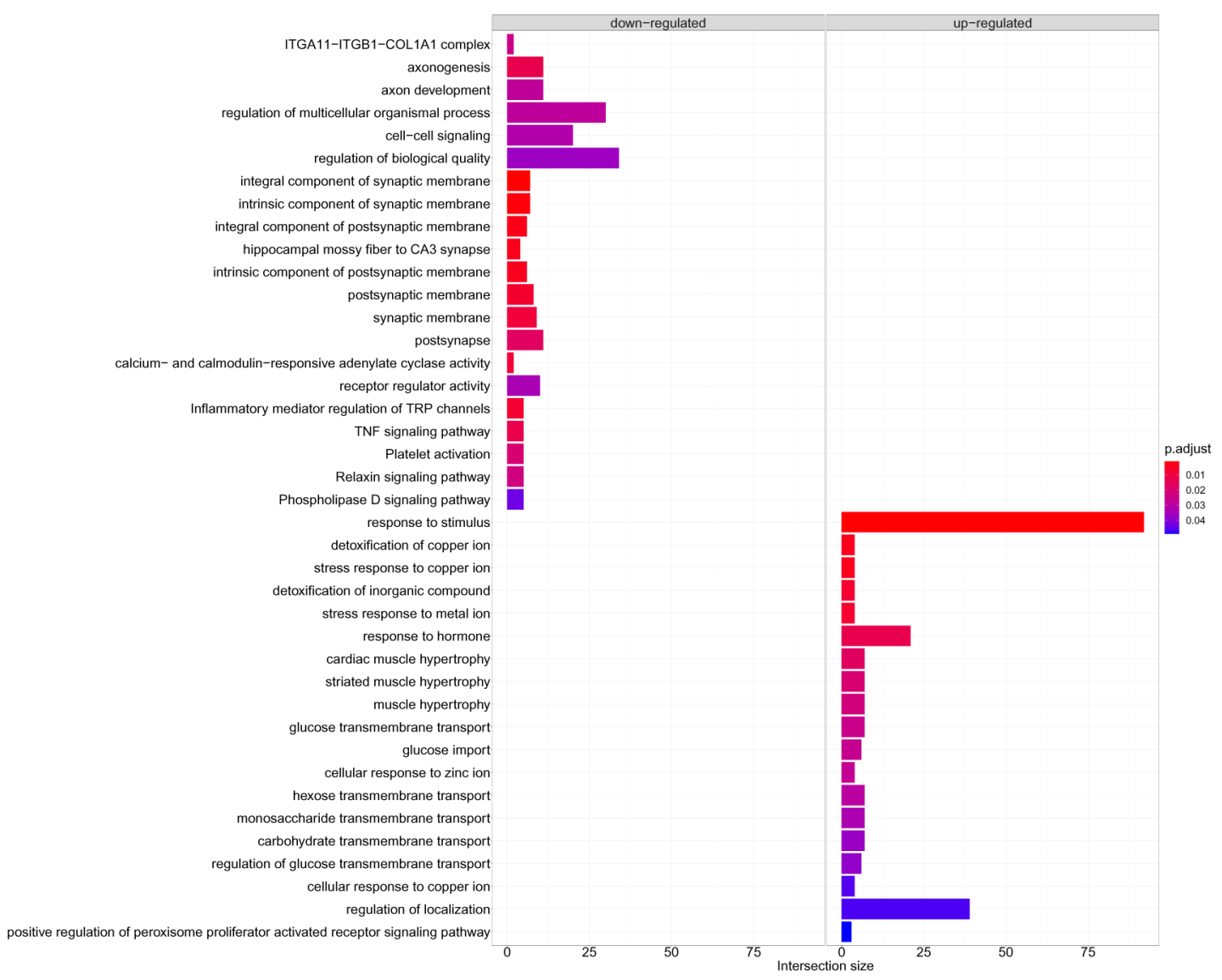

Figure 5. Bar plots of g:Profiler enrichment results using enrichplot.

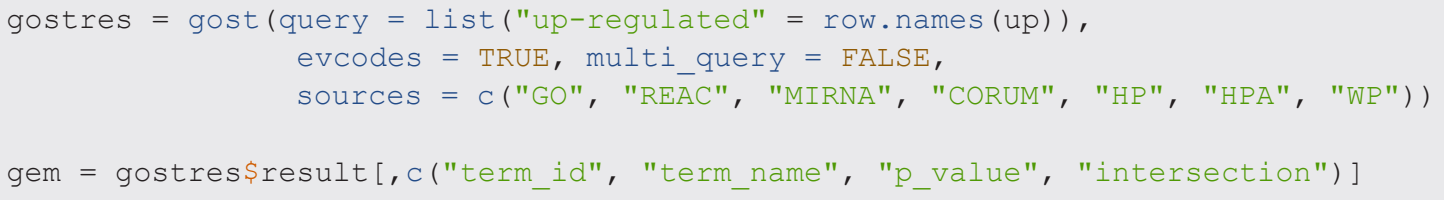

In the EnrichmentMap the user can set the "Analysis Type" parameter as Generic/gProfiler and upload the required files: GEM file with enrichment results (input field "Enrichments") and GMT file that defines the annotations (input field "GMT"). Both of these files have to include gene identifiers from the same namespace for the EnrichmentMap to work.

The GMT files used by g:Profiler are downloadable from the web page under the "Data sources" section. Only the GMT files of KEGG and Transfac are not available as the sharing is restricted by data source licenses.

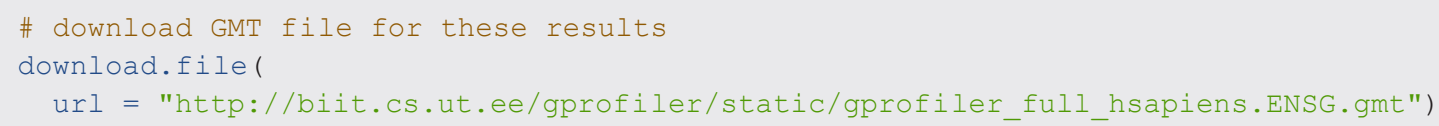




\section{Reproducibility}

The demand for better reproducibility of computational analyses is constantly growing ${ }^{30}$. In bioinformatics analysis, many different tools and databases are combined in order to detect relevant findings. This adds an extra layer of complexity which often leads to reproducibility issues. Because of this, since 2011 all the past releases of g:Profiler are maintained and kept usable to ensure reproducibility and transparency of enrichment analysis results. The users can cite the exact extract of the annotation database and the state of the implementation by stating the version number in their research. In gprofiler2, this is available, along with other query information, from the metadata of gost enrichment results:

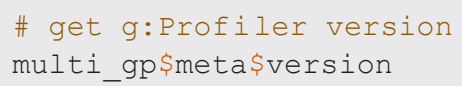

The version number notes that the results were obtained using the state of the database that includes data from Ensembl release 99, Ensembl Genomes release 46 and WormBase ParaSite release 14, and the g:Profiler codebase with the Git revision number f929183. The version number together with the details of applied parameters (available from multi_gp\$meta\$query_metadata) is enough to reproduce the results.

In order to reproduce the results obtained with a specific version, one can change the data version using the function set_base_url:

$$
\text { set_base_url("http://biit.cs.ut.ee/gprofiler_archive3/e99_eg46_p14") }
$$

All the past versions and their URLs are available at https://biit.cs.ut.ee/gprofiler/page/archives. gprofiler2 works with versions e94_eg41_p11 and higher, earlier versions are still accessible using the deprecated R package gProfileR.

Function set base url also gives access to the most recent developments and data updates of g:Profiler available at the Beta version:

set_base_url("http://biit.cs.ut.ee/gprofiler_beta")

In order to determine the current $\mathrm{g}$ :Profiler URL used for the analysis one can use the function get_base_url:

get_base_url ()

\#\# [1] "http://biit.cs.ut.ee/gprofiler_beta"

\section{Conclusion}

We presented the gprofiler $2 \mathrm{R}_{\text {package }}^{8}$ that is one of the programmatic access points to the widely used g:Profiler web toolset for gene list functional enrichment analysis and identifier conversion. This package enables effective integration of $\mathrm{g}$ :Profiler functionalities in various bioinformatics pipelines and tools written in $\mathrm{R}$ without the need of searching and downloading several data files. The suite of functions in gprofiler 2 are implemented with the importance of analysis reproducibility and interoperability with other tools in mind. In addition, the package provides a way to easily create or customise the enrichment plots using the existing visualisation packages in R. For the researchers who prefer to perform their computational analysis pipelines through the web we have wrapped the gprofiler2 package as a tool for the Galaxy platform ${ }^{31}$.

It is important to note that using gprofiler 2 for functional enrichment analysis is not limited to the use case of differential gene expression analysis. The package is useful whenever there is a set of genes/proteins/SNPs the user wants to characterise with biological functions or to convert to another namespace.

Data availability

All data underlying the results are available as part of the article and no additional source data are required.

\section{Software availability \\ R package gprofiler2 is available from CRAN: https://cran.r-project.org/package=gprofiler2.}


Source code available from: https://gl.cs.ut.ee/biit/r-gprofiler2.

Archived source code at time of publication: https://doi.org/10.5281/zenodo. $3919795^{8}$.

License: GNU General Public License v2.0.

Author information

LK, UR and IK implemented the package. LK and HP wrote the article. HP and JV supervised the development.

Acknowledgments

We would like to thank Kaur Alasoo for critically reading the manuscript.

1. Reimand J, Kull M, Peterson $\mathrm{H}$, et al:: g:profiler - a web-based toolset for functional profiling of gene lists from large-scale experiments. Nucleic Acids Res. 2007; 35(suppl_2): W193-W200. PubMed Abstract | Publisher Full Text | Free Full Text

2. Raudvere U, Kolberg L, Kuzmin I, et al:: g:profiler: a web server for functional enrichment analysis and conversions of gene lists (2019 update). Nucleic Acids Res. 2019; 47(W1): W191-W198. PubMed Abstract | Publisher Full Text | Free Full Text

3. Ashburner M, Ball CA, Blake JA, et al:: Gene Ontology: Tool for the Unification of Biology. The Gene Ontology Consortium. Nat Genet. 2000; 25(1): 25-29.

PubMed Abstract | Publisher Full Text | Free Full Text

4. Kanehisa M, Sato Y, Furumichi M, et al.: New approach for understanding genome variations in kegg. Nucleic Acids Res. 2019; 47(D1): D590-D595.

PubMed Abstract | Publisher Full Text | Free Full Text

5. Fabregat $A$, Jupe $S$, Matthews $L$, et al:: The reactome pathway knowledgebase. Nucleic Acids Res. 2018; 46(Database issue): D649-D655.

PubMed Abstract | Publisher Full Text | Free Full Text

6. Yates AD, Achuthan P, Akanni W, et al.: Ensembl 2020. Nucleic Acids Res. 2020; 48(D1): D682-D688.

PubMed Abstract | Publisher Full Text | Free Full Text

7. Howe KL, Bolt BJ, Shafie M, et al:: Wormbase parasite- a comprehensive resource for helminth genomics. Mol Biochem Parasitol. 2017; 215: 2-10

PubMed Abstract | Publisher Full Text | Free Full Text

8. Kolberg L, Raudvere U, Kuzmin I, et al.: gprofiler2 R package (version 0.1.9). 2020

http://www.doi.org/10.5281/zenodo.3919795

9. Alexa A, Rahnenfuhrer J: topGO: Enrichment Analysis for Gene Ontology. R package version 2.38.1. 2019. Reference Source

10. Yu G, Wang LG, Han Y, et al: clusterProfiler: an R Package for Comparing Biological Themes Among Gene Clusters. OMICS. 2012; 16(5): 284-287.

PubMed Abstract | Publisher Full Text | Free Full Text

11. Uyar B, Yusuf D, Wurmus R, et al:: Rcas: an rna centric annotation system for transcriptome-wide regions of interest. Nucleic Acids Res. 2017; 45(10): e91.

PubMed Abstract | Publisher Full Text | Free Full Text

12. Sokolowski D, Faykoo-Martinez M, Wilson M, et al.: scMappR: Single Cell Mapper. $R$ package version 0.1.1. 2020.

Reference Source

13. Valdeolivas A, Turei D, Gabor A: Omnipathr: Import omnipath network. Bioconductor Package. 2019

Publisher Full Text

14. Lang DT: RCurl: General Network (HTTP/FTP/...) Client Interface for R. 2020.

Reference Source

15. Wickham H: ggplot2: Elegant Graphics for Data Analysis. Springer-Verlag New York. 2016. Reference Source

16. Sievert C: Interactive Web-Based Data Visualization with R plotly, and shiny. Chapman and Hall/CRC. 2020. Reference Source

17. Slenter DN, Kutmon M, Hanspers K, et al.: Wikipathways: a multifaceted pathway database bridging metabolomics to other omics research. Nucleic Acids Res. 2018; 46(D1): D661-D667. PubMed Abstract | Publisher Full Text | Free Full Text

18. Chou $\mathrm{CH}$, Shrestha $\mathrm{S}$, Yang $\mathrm{CD}$, et al.: mirtarbase update 2018: a resource for experimentally validated microrna-target interactions. Nucleic Acids Res. 2018; 46(D1): D296-D302.

PubMed Abstract | Publisher Full Text | Free Full Text

19. Matys V, Kel-Margoulis OV, Fricke E, et al:: TRANSFAC $₫$ and its module TRANSCompel $囚$ : transcriptional gene regulation in eukaryotes. Nucleic Acids Res. 2006; 34(Database issue): D108-D110.

PubMed Abstract | Publisher Full Text | Free Full Text

20. Uhlén M, Fagerberg L, Hallström BM, et al: Proteomics. Tissuebased Map of the Human Proteome. Science. 2015; 347(6220): 1260419.

PubMed Abstract | Publisher Full Text

21. Ruepp A, Waegele B, Lechner M, et al:: Corum: the comprehensive resource of mammalian protein complexes-2009. Nucleic Acids Res. 2010; 38(suppl_1): D497-D501.

PubMled Abstract | Publisher Full Text | Free Full Text

22. Köhler S, Carmody L, Vasilevsky N, et al.: Expansion of the human phenotype ontology (hpo) knowledge base and resources. Nucleic Acids Res. 2019; 47(D1): D1018-D1027. PubMed Abstract | Publisher Full Text | Free Full Text

23. Love MI, Anders S, Kim V, et al.: RNA-Seq workflow: gene-level exploratory analysis and differential expression [version 1; peer review: 2 approved]. F1000Res. 2015; 4. PubMed Abstract | Publisher Full Text | Free Full Text

24. Love MI, Huber W, Anders S: Moderated estimation of fold chang and dispersion for rna-seq data with deseq2. Genome Biol. 2014; 15: 550 .

PubMed Abstract | Publisher Full Text | Free Full Text

25. Himes $B E$, Jiang $X$, Wagner $P$, et al: RNA-Seq Transcriptome Profiling Identifies CRISPLD2 as a Glucocorticoid Responsive Gene that Modulates Cytokine Function in Airway Smooth Muscle Cells. PLoS One. 2014; 9(6): e99625. PubMed Abstract | Publisher Full Text | Free Full Text

26. Subramanian A, Tamayo P, Mootha VK, et al:: Gene set enrichment analysis: a knowledge- based approach for interpreting genomewide expression profiles. Proc Natl Acad Sci U S A. 2005; 102(43): $15545-15550$

PubMed Abstract | Publisher Full Text | Free Full Text

27. Piñero J, Ramírez-Anguita JM, Saüch-Pitarch J, et al.: The disgenet knowledge platform for disease genomics: 2019 update. Nucleic Acids Res. 2020; 48(D1): D845-D855.

PubMed Abstract | Publisher Full Text | Free Full Text

28. Yu S: enrichplot: Visualization of Functional Enrichment Result. 2019

Reference Source

29. Merico D, Isserlin R, Stueker O, et al.: Enrichment map: a network- 
based method for gene-set enrichment visualization and interpretation. PLoS One. 2010; 5(11): e13984. PubMed Abstract | Publisher Full Text | Free Full Text

30. Kulkarni N, Alessandrì L, Panero R, et al.: Reproducible bioinformatics project: a community for reproducible bioinformatics analysis pipelines. BMC Bioinformatics. 2018;
19(Suppl 10): 349

PubMed Abstract | Publisher Full Text | Free Full Text

31. Afgan E, Baker D, Batut B, et al.: The galaxy platform for accessible, reproducible and collaborative biomedical analyses: 2018 update. Nucleic Acids Res. 2018; 46(W1): W537-W544. PubMed Abstract | Publisher Full Text | Free Full Text 


\section{Open Peer Review}

\section{Current Peer Review Status: ?}

\section{Version 1}

Reviewer Report 01 September 2020

https://doi.org/10.5256/f1000research.27533.r67235

(C) 2020 Willighagen E. This is an open access peer review report distributed under the terms of the Creative Commons Attribution License, which permits unrestricted use, distribution, and reproduction in any medium, provided the original work is properly cited.

\section{Egon L. Willighagen}

Department of Bioinformatics-BiGCaT, NUTRIM, Maastricht University, Maastricht, The Netherlands

The article describes an R package (gprofiler2) that wraps around the REST interface of a g:Profiler web service. The paper outlines the method used to access the API, and explains that providing an R API makes it easier to integrate with the functionality of other R packages. The description of the software is sufficient and complementary to the source code repository (and backed by a Zenodo archive). The examples given nicely demonstrate the functionality and I tried many of them in rstudio (some comments below). The visualisation, as is most functionality, is inherited from the website, and as such benefits from a larger user base.

The choice of the combination of having the code base under version control, archiving on Zenodo, and distribution via CRAN shows high-quality standards. A test suite, unfortunately, is missing, and adding it is recommended. Furthermore, it could be interesting to explore curl as a replacement of RCurl, as I had trouble with SSL certificates with the RCurl package on Windows (but not essential).

I can build the package from source. When applying a regular CRAN check on the package I get a couple of warnings and notes, but none that are affecting this paper. I left two source code suggestions: https://github.com/egonw/r-gprofiler2/pull/1.

I also like to note that I found out that the interactive plot does not seem to work in RStudio: I only get an empty window.

Regarding the content of the article, I have the following questions. First, the argument that the computation is done on a central server as an advantage depends on the use case and is not generally true. For example, it requires all information to be shared with the server, which is not possible for everyone that may be interested in the server. As such, one could imagine having a Docker with the webservice and method parameters in the $\mathrm{R}$ package to interact with servers with a different domain or IP address. This is, however, just a future feature request. 
Second, regarding the data used in the functionality, I have three questions. How is the ordering of pathways in for KEGG, Reactome, and WikiPathways defined? For Reactome, I can imagine their own hierarchy is used. What about KEGG and WikiPathways? Is the Pathway Ontology used for the latter? A similar question applies when using custom GMT files? The ordering is then defined by the other in the file, I assume? Finally, in the Reproducibility section, but it is not clear to me why version information is not given for the data GO, KEGG, Reactome, etc. I would strongly suggest providing that information too. I would suggest clarifying the first two in the manuscript, but the last one may require additional functionality. Alternatively, the article should be clear that version information is only given for a subset of used resources.

A third topic I like to ask about is the following: If $\mathrm{g}$ :Profile is an ELIXIR RIR, have you consider adding the option to use the Identifiers.org prefixes, e.g. "wikipathways" in addition to "WP"?

Fourth, the "Use case" uses packages that need to be installed. The keep symmetry with the description of gprofiler 2 itself, please consider adding install instructions for these Bioconductor packages.

Last, I collected a number of small textual suggestions and have annotated these with hypothes.is: https://hyp.is/rE4tRurJEeqMHW_RYqO0xA/f1000research.com/articles/9-709.

These suggestions include a number of title fixes in the bibliography where article titles are all converted to lower case, which looks weird for names of tools.

Is the rationale for developing the new software tool clearly explained?

Yes

Is the description of the software tool technically sound?

Yes

Are sufficient details of the code, methods and analysis (if applicable) provided to allow replication of the software development and its use by others?

Yes

Is sufficient information provided to allow interpretation of the expected output datasets and any results generated using the tool?

Yes

Are the conclusions about the tool and its performance adequately supported by the findings presented in the article?

Yes

Competing Interests: I am (co-)author if similar analysis R packages and webservice (BridgeDbR, rWikiPathways, BridgeDb webservice, WikiPathways). There is no active collaboration, but we are member of ELIXIR and BridgeDb is another Recommended Interoperability Resource. These interests are not direct and the final decision on this paper does not affect our own research. 
Reviewer Expertise: R packages, identifier mapping, biological pathway resources, metabolomics, cheminformatics

I confirm that I have read this submission and believe that I have an appropriate level of expertise to confirm that it is of an acceptable scientific standard, however I have significant reservations, as outlined above.

Author Response 15 Sep 2020

Hedi Peterson, University of Tartu, Estonia

We thank the reviewer for thorough reading of the manuscript and for the suggestions to improve the gprofiler 2 package and the g:Profiler tool itself. Reviewer's comments are in bold followed by our responses in regular font.

The choice of the combination of having the code base under version control, archiving on Zenodo, and distribution via CRAN shows high-quality standards. A test suite, unfortunately, is missing, and adding it is recommended.

We agree that testing is an important part of software development. However, since the main functionality of the gprofiler2 R package is to wrap POST requests to g:Profiler API, which has an extensive test suite, then we decided to not include additional tests to the package. Nevertheless, we use the R CMD checks for the package every time we submit a new version to CRAN.

Furthermore, it could be interesting to explore curl as a replacement of RCurl, as I had trouble with SSL certificates with the RCurl package on Windows (but not essential).

Thank you for the suggestion. The decision to use the RCurl package was made in 2012 when development started for the first version of the $g$ :Profiler package. Replacing this dependency now can potentially cause issues for current users and external pipelines. Therefore, at the moment, we will keep this dependency as is, but we might consider the change in future updates. We are aware of the issues related to SSL certificates on Windows and due to that we provide access to g:Profiler both with and without SSL.

I can build the package from source. When applying a regular CRAN check on the package I get a couple of warnings and notes, but none that are affecting this paper. I left two source code suggestions: https://github.com/egonw/r-gprofiler2/pull/1.

Thank you for the suggestions, we included the suggested changes.

I also like to note that I found out that the interactive plot does not seem to work in RStudio: I only get an empty window.

It appears that this is a known issue with rendering interactive plotly images in the latest RStudio version (see https://github.com/ropensci/plotly/issues/1712 and https://github.com/rstudio/rstudio/issues/7507, for example). One of the publicly proposed 
solutions is to reinstall RStudio. We hope this solves the interactivity issue.

Regarding the content of the article, I have the following questions.

First, the argument that the computation is done on a central server as an advantage depends on the use case and is not generally true. For example, it requires all information to be shared with the server, which is not possible for everyone that may be interested in the server. As such, one could imagine having a Docker with the webservice and method parameters in the $R$ package to interact with servers with a different domain or IP address. This is, however, just a future feature request.

We agree that using a central server is problematic for users who cannot share their data. However, centralising computations enables us to maintain the $\mathrm{g}$ :Profiler ecosystem better and guarantees that the results from different access points (web tool, R package, Python package, Galaxy tools, custom API requests) remain consistent, which is beneficial for the users. To alleviate data sharing issue we do not store the input gene lists in g:Profiler unless the user explicitly requests their data to be stored for future access/reference via dedicated short links. This information is provided at the footer of the $\mathrm{g}$ :Profiler web page, and will be included within the next version of the R package (0.2.1). In addition, we added the following sentences (underlined) to the manuscript:

"Relying on the central API also simplifies the maintenance of the g:Profiler interfaces and enables the $\mathrm{R}$ users to get access to the most up-to-date data without having to download the heavy annotation data files to their own devices. At the same time, g:Profiler respects users' privacy and does not store input gene lists unless the user explicitly requests their data to be stored for future reference via dedicated short links (see section "Sending analysis from R to g:Profiler web interface")."

"In this case, the variable multi_gp_link is a character string that corresponds to a stable short-link to these enrichment results: https://biit.cs.ut.ee/gplink/l/0wgtcERnQT. We also note that the input gene lists will be stored in a database to provide short-link access."

Second, regarding the data used in the functionality, I have three questions. How is the ordering of pathways in for KEGG, Reactome, and WikiPathways defined? For Reactome, I can imagine their own hierarchy is used. What about KEGG and WikiPathways? Is the Pathway Ontology used for the latter?

We use the order provided by the corresponding data source for all three of them. For example, pathways from KEGG are provided in the order of their identifiers, but pathways from Reactome are ordered by the name of the pathway term. We do not currently use the Pathway Ontology for WikiPathways. Thus, the only source for which we use hierarchical ontology order in the Manhattan plot is Gene Ontology, but the x-axis positions are fixed for all of the sources. This means that the terms in the Manhattan plot do not change the position along the $x$-axis when the input query changes.

A similar question applies when using custom GMT files? The ordering is then defined by the other in the file, I assume? 
Yes, in case of custom GMT files, the order of the terms in the Manhattan plot is defined by their order in the GMT file. We included the following sentence (underlined) to the manuscript to clarify this:

"The custom data source results can also be plotted using the Manhattan plots (Figure 3). In this case, the term position on the x-axis is defined by the order in the GMT file."

Finally, in the Reproducibility section, but it is not clear to me why version information is not given for the data GO, KEGG, Reactome, etc. I would strongly suggest providing that information too. I would suggest clarifying the first two in the manuscript, but the last one may require additional functionality. Alternatively, the article should be clear that version information is only given for a subset of used resources.

Historically, g:Profiler versioning schema is based on Ensembl releases because we update our database according to their update schedule (with a time lag). The version fixes the exact extract of g:Profiler annotation database to give a point of reference for $\mathrm{g}$ :Profiler enrichment results and is not intended to cover all the numerous data source versions. However, we agree that the source specific version information might be useful for the users and it is available from the g:Profiler's web page (under the "Data sources" section there is a link "Show data versions"). We included this information to the revised manuscript. Furthermore, based on this suggestion, we also added a new function get_version_info(organism) to the R package that will enable users to obtain the versions of all the data sources used for the set $\mathrm{g}$ :Profiler version and organism. The function is already in the source code repository https://gl.cs.ut.ee/biit/r-gprofiler2, but currently works only for the Beta version, i.e. if set_base_url("http://biit.cs.ut.ee/gprofiler_beta"). This functionality will be included in the next CRAN release of the gprofiler2 $\mathrm{R}$ package (0.2.1).

The amendments in the section "Reproducibility" to clarify the g:Profiler version info are underlined in the excerpt below.

"The g:Profiler specific version number notes that the results were obtained using the state of the database that includes data from Ensembl release 99, Ensembl Genomes release 46 and WormBase ParaSite release 14, among other sources, and the g:Profiler codebase with the Git revision number f929183. The version number together with the details of applied parameters (available from multi_gp\$meta\$query_metadata) is enough to reproduce the enrichment results in $\mathrm{g}$ :Profiler. A more detailed information about the data source versions in a given g:Profiler version is available from the g:Profiler web page https://biit.cs.ut.ee/gprofiler under the link "Show data versions" in the "Data sources" section."

A third topic I like to ask about is the following: If g:Profile is an ELIXIR RIR, have you consider adding the option to use the Identifiers.org prefixes, e.g. "wikipathways" in addition to "WP"?

We have not considered the option to use the identifiers.org prefixes at the moment. Currently all the terms are linked inside the g:Profiler toolset and no external links are provided. However, we thank the reviewer for this suggestion and we will consider it for 
further g:Profiler releases.

Fourth, the "Use case" uses packages that need to be installed. The keep symmetry with the description of gprofiler 2 itself, please consider adding install instructions for these Bioconductor packages.

We included the suggested installation instructions in the revised version of the manuscript.

Last, I collected a number of small textual suggestions and have annotated these with hypothes.is: https://hyp.is/rE4tRurJEeqMHW_RYqO0xA/f1000research.com/articles/9709. These suggestions include a number of title fixes in the bibliography where article titles are all converted to lower case, which looks weird for names of tools.

Thank you very much for noticing the issues from bibliography parsing. We updated the manuscript according to the suggestions.

Competing Interests: No competing interests were disclosed.

The benefits of publishing with F1000Research:

- Your article is published within days, with no editorial bias

- You can publish traditional articles, null/negative results, case reports, data notes and more

- The peer review process is transparent and collaborative

- Your article is indexed in PubMed after passing peer review

- Dedicated customer support at every stage

For pre-submission enquiries, contact research@f1000.com 